ANNALES

POLONICI MATHEMATICI

$84.3(2004)$

\title{
Existence results for a class of quasilinear integrodifferential equations of Volterra-Hammerstein type with nonlinear boundary conditions
}

\author{
by ZuOdONG YANG (Nanjing)
}

\begin{abstract}
The existence of a solution for a class of quasilinear integrodifferential equations of Volterra-Hammerstein type with nonlinear boundary conditions is established. Such equations occur in the study of the $p$-Laplace equation, generalized reactiondiffusion theory, non-Newtonian fluid theory, and in the study of turbulent flows of a gas in a porous medium. The results are obtained by using upper and lower solutions, and extend some previously known results.
\end{abstract}

In this paper we study existence results for the integrodifferential equation

$$
\left(\Phi_{m}\left(u^{\prime}\right)\right)^{\prime}=f\left(t, u, T_{1} u, T_{2} u, u^{\prime}\right), \quad t \in I=[0,1],
$$

subject to one of the following boundary conditions:

$$
g\left(u(0), u(1), u^{\prime}(0), u^{\prime}(1)\right)=0, \quad h(u(0))=u(1),
$$

Or

$$
p\left(u(0), u^{\prime}(0)\right)=0=q\left(u(0), u^{\prime}(0), u(1), u^{\prime}(1)\right)
$$

or

$$
r\left(u(1), u^{\prime}(1)\right)=0=w\left(u(0), u^{\prime}(0), u(1), u^{\prime}(1)\right)
$$

where

$$
T_{1} u(t)=\psi_{1}(t)+\int_{0}^{t} K_{1}(t, s) u(s) d s, \quad T_{1} u(t)=\psi_{2}(t)+\int_{0}^{t} K_{2}(t, s) u(s) d s,
$$

2000 Mathematics Subject Classification: Primary 34B15.

Key words and phrases: integrodifferential equation of Volterra-Hammerstein type; nonlinear boundary value problem; upper and lower solutions.

Research supported by the Science Foundation of Nanjing Normal University (No. 2003SXXXGQ2B37), the Science Foundation of 211 Engineering and the Science Foundation of Jiangsu Province Educational Department (No. 04KJB110062). 
$K_{i} \in C\left([0,1] \times[0,1], \mathbb{R}^{+}\right), \psi_{i} \in C([0,1], \mathbb{R}), i=1,2, f:[0,1] \times \mathbb{R}^{4} \rightarrow \mathbb{R}$ is a continuous function, and $\Phi_{m}(s)=|s|^{m-2} s$ for $m>1$. Equations of the above form are mathematical models occurring in the study of the $m$-Laplace equation, in generalized reaction-diffusion theory ([6]), non-Newtonian fluid theory, and in the study of turbulent flows of a gas in a porous medium ([4]). In the non-Newtonian fluid theory, the quantity $m$ is a characteristic of the medium. Media with $m>2$ are called dilatant fluids and those with $m<2$ are called pseudoplastics. If $m=2$, they are Newtonian fluids.

The equation

$$
\left(\Phi_{m}\left(u^{\prime}\right)\right)^{\prime}=f\left(t, u, u^{\prime}\right), \quad t \in I=[0,1],
$$

with various boundary conditions has been studied by many authors (see $[1-4,6,8-15]$ and references therein). On the contrary, it seems that little is known about problems (1)-(2), (1)-(3), and (1)-(4). Our results were motivated by the papers $[1,2,5,7]$ which studied periodic and Neumann nonlinear boundary conditions for equation (5). When $p=2$, some related results have been obtained in $[5,7]$. Our results extend those of $[1,2,5,7]$.

Definition 1. A function $\alpha \in C^{1}[0,1]$ with $\Phi_{m}\left(\alpha^{\prime}\right) \in C^{1}[0,1]$ is called a lower solution of $(5)$ on $I=[0,1]$ if

$$
\left(\Phi_{m}\left(\alpha^{\prime}\right)\right)^{\prime} \geq f\left(t, \alpha, \alpha^{\prime}\right) \quad \text { for } t \in I .
$$

Likewise, $\beta \in C^{1}[0,1]$ with $\Phi_{m}\left(\beta^{\prime}\right) \in C^{1}[0,1]$ is an upper solution of (5) on $I$ if

$$
\left(\Phi_{m}\left(\beta^{\prime}\right)\right)^{\prime} \leq f\left(t, \beta, \beta^{\prime}\right) \quad \text { for } t \in I .
$$

In what follows we shall assume that

$$
\alpha(t) \leq \beta(t), \quad t \in I .
$$

For $\alpha, \beta \in C(I), \alpha \leq \beta$, we define the set

$$
E=\left\{u \in C^{1}(I) \mid \alpha(t) \leq u(t) \leq \beta(t), \forall t \in I\right\} .
$$

In the following theorems we will use the following hypotheses:

$\left(\mathrm{H}_{1}\right) \quad f$ is a continuous function in $\Omega=\left\{(t, y, z) \mid 0 \leq t \leq 1,(y, z) \in \mathbb{R}^{2}\right\}$. $\left(\mathrm{H}_{2}\right) \quad f(t, y, z)$ satisfies the Nagumo condition in $E$, i.e. there exists a function $\Psi:[0, \infty) \rightarrow[0, \infty)$ with $1 / \Psi$ integrable on every bounded interval $(a, b) \subset[0, \infty)$, such that

$$
|f(t, y, z)| \leq \Psi(|z|) \quad \text { for }(t, y) \in E, z \in \mathbb{R}
$$

where $\Psi$ satisfies

$$
\int_{0}^{\infty} \Phi_{m}^{-1}(u) / \Psi\left(\Phi_{m}^{-1}(u)\right) d u=\infty .
$$

From $[11,15]$, we have the following theorem: 
THEOREM 1. Let $\alpha, \beta \in C^{1}[0,1]$ be lower and upper solutions of (5), respectively, with $\alpha \leq \beta$ in $I$. Assume that hypotheses $\left(\mathrm{H}_{1}\right)-\left(\mathrm{H}_{2}\right)$ are satisfied. Then for any $\alpha(0) \leq A \leq \beta(0), \alpha(1) \leq B \leq \beta(1)$ there exists a solution $u$ of the boundary value problem

$$
\left(\Phi_{m}\left(u^{\prime}\right)\right)^{\prime}=f\left(t, u, u^{\prime}\right), \quad u(0)=A, \quad u(1)=B,
$$

satisfying $\alpha(t) \leq u(t) \leq \beta(t)$ on $[0,1]$.

Before stating the main result on existence of solutions for problems (1)-(2), (1)-(3), and (1)-(4), we give the following

Definition 2. A function $\alpha \in C^{1}[0,1]$ with $\Phi_{m}\left(\alpha^{\prime}\right) \in C^{1}[0,1]$ is called a lower solution of $(1)$ on $[0,1]$ if

$$
\left(\Phi_{m}\left(\alpha^{\prime}\right)\right)^{\prime} \geq f\left(t, \alpha, T_{1} \alpha, T_{2} \alpha, \alpha^{\prime}\right) \quad \text { for } t \in I .
$$

Likewise, $\beta \in C^{1}[0,1]$ with $\Phi_{m}\left(\beta^{\prime}\right) \in C^{1}[0,1]$ is an upper solution of (1) on $[0,1]$ if

$$
\left(\Phi_{m}\left(\beta^{\prime}\right)\right)^{\prime} \leq f\left(t, \beta, T_{1} \beta, T_{2} \beta, \beta^{\prime}\right) \quad \text { for } t \in I .
$$

Moreover, we define the following sets:

$$
\begin{aligned}
& F=\{(y, z, u, v)|| \alpha(0) \leq y \leq \beta(0), \alpha(1) \leq z \leq \beta(1), u, v \in \mathbb{R}\} ; \\
& G=\{g=g(y, z, u, v) \in C(F) \mid g \text { is nondecreasing in } u, \text { nonincreasing in } v, \\
& \left.\quad \text { and } g\left(\alpha(0), \alpha(1), \alpha^{\prime}(0), \alpha^{\prime}(1)\right) \geq 0 \geq g\left(\beta(0), \beta(1), \beta^{\prime}(0), \beta^{\prime}(1)\right)\right\} ; \\
& H=\{h \mid h:[\alpha(0), \beta(0)] \rightarrow[\alpha(1), \beta(1)] \text { is a homeomorphism, } \\
& \text { and } h(\alpha(0))=\alpha(1), h(\beta(0))=\beta(1)\} ;
\end{aligned}
$$$$
P=\{p=p(s, t) \mid p:[\alpha(0), \beta(0)] \times \mathbb{R} \rightarrow \mathbb{R} \text { is continuous and nonincreasing }
$$$$
\text { in } \left.t \text { and } p\left(\alpha(0), \alpha^{\prime}(0)\right) \leq 0 \leq p\left(\beta(0), \beta^{\prime}(0)\right)\right\} ;
$$$$
\Gamma=\{(s, t, u, v) \mid \alpha(0) \leq s \leq \beta(0), \alpha(1) \leq u \leq \beta(1), t, v \in \mathbb{R}\} ;
$$$$
Z(p)=\{(s, t) \mid p(s, t)=0, \alpha(0) \leq s \leq \beta(0), t \in \mathbb{R}\} ;
$$$$
Q=\{q=q(s, t, u, v) \in C(\Gamma) \mid q \text { is nondecreasing in } v \text {, and }
$$$$
\left.q\left(s, t, \alpha(1), \alpha^{\prime}(1)\right) \leq 0 \leq q\left(s, t, \beta(1), \beta^{\prime}(1)\right) \text { for }(s, t) \in Z(p)\right\} ;
$$$$
Z(r)=\{(u, v) \mid r(u, v)=0, \alpha(1) \leq u \leq \beta(1), v \in \mathbb{R}\} ;
$$$$
R=\{r=r(u, v) \mid r:[\alpha(1), \beta(1)] \times \mathbb{R} \rightarrow \mathbb{R} \text { is continuous, nondecreasing in } v,
$$$$
\text { and } \left.r\left(\alpha(1), \alpha^{\prime}(1)\right) \leq 0 \leq r\left(\beta(1), \beta^{\prime}(1)\right)\right\} ;
$$

$W=\{w=w(s, t, u, v) \in C(\Gamma) \mid w$ is nonincreasing in $t$, and

$$
\left.w\left(\alpha(0), \alpha^{\prime}(0), u, v\right) \leq 0 \leq w\left(\alpha(1), \alpha^{\prime}(1), u, v\right) \text { for }(u, v) \in Z(r)\right\} .
$$


In what follows we impose the following conditions on (1):

$\left(\mathrm{H}_{3}\right) \quad f(t, u, v, w, z)$ is nonincreasing in $v$ and in $w$.

$\left(\mathrm{H}_{4}\right) \quad f \in C\left([0,1] \times \mathbb{R}^{4}, \mathbb{R}\right)$ and there exists a continuous function $h$ : $[0, \infty) \rightarrow[0, \infty)$ such that

$$
|f(t, u, v, w, z)| \leq h(|z|) \quad \text { for }(t, u, v, w, z) \in \Omega,
$$

where $\Omega=\left\{(t, u, v, w, z) \in I \times \mathbb{R}^{3}:|u| \leq r_{1},|v| \leq r_{2},|w| \leq r_{3}\right.$, $z \in \mathbb{R}\}$ for some $r_{1}, r_{2}, r_{3}>0$, and also that

$$
\int_{0}^{\infty} \Phi_{m}^{-1}(u) / h\left(\Phi_{m}^{-1}(u)\right) d u=\infty .
$$

Now, we can prove our main results.

THEOREM 2. Let $\alpha, \beta \in C^{1}[0,1]$ be lower and upper solutions of (1), respectively, with $\alpha \leq \beta$ on $I=[0,1]$. Assume that hypotheses $\left(\mathrm{H}_{3}\right)-\left(\mathrm{H}_{4}\right)$ are satisfied. Then for any $\alpha(0) \leq A \leq \beta(0), \alpha(1) \leq B \leq \beta(1)$ there exists a solution $u$ of the boundary value problem

$$
\left(\Phi_{m}\left(u^{\prime}\right)\right)^{\prime}=f\left(t, u, T_{1} u, T_{2} u, u^{\prime}\right), \quad u(0)=A, \quad u(1)=B,
$$

satisfying $\alpha(t) \leq u(t) \leq \beta(t)$ on $[0,1]$.

Proof. Let $u_{0}(t)=\beta(t)$. Then

$$
\begin{aligned}
f\left(t, \alpha(t),\left[T_{1} u_{0}\right](t),\left[T_{2} u_{0}\right](t), \alpha^{\prime}(t)\right) & \leq f\left(t, \alpha,\left[T_{1} \alpha\right](t),\left[T_{2} \alpha\right](t), \alpha^{\prime}(t)\right) \\
& \leq\left(\Phi_{m}\left(\alpha^{\prime}\right)\right)^{\prime}, \\
f\left(t, \beta(t),\left[T_{1} u_{0}\right](t),\left[T_{2} u_{0}\right](t), \beta^{\prime}(t)\right) & \geq\left(\Phi_{m}\left(\beta^{\prime}\right)\right)^{\prime}, \quad t \in I=[0,1] .
\end{aligned}
$$

By Theorem 1, there exists a solution $u_{1}$ of the boundary value problem

$$
\left(\Phi_{m}\left(u^{\prime}\right)\right)^{\prime}=f\left(t, u, T_{1} u_{0}, T_{2} u_{0}, u^{\prime}\right), \quad u(0)=A, \quad u(1)=B,
$$

satisfying $\alpha(t) \leq u_{1}(t) \leq \beta(t)=u_{0}(t)$ on $[0,1]$.

We now consider the problem

$$
\left(\Phi_{m}\left(u^{\prime}\right)\right)^{\prime}=f\left(t, u, T_{1} u_{1}, T_{2} u_{1}, u^{\prime}\right), \quad u(0)=A, \quad u(1)=B .
$$

Clearly,

$$
f\left(t, \alpha, T_{1} u_{1}, T_{2} u_{1}, \alpha^{\prime}\right) \leq\left(\Phi_{m}\left(\alpha^{\prime}\right)\right)^{\prime}
$$

and

$$
f\left(t, u_{1}, T_{1} u_{1}, T_{2} u_{1}, u_{1}^{\prime}\right) \geq f\left(t, u_{1}, T_{1} u_{0}, T_{1} u_{0}, u_{1}^{\prime}\right)=\left(\Phi_{m}\left(u_{1}^{\prime}\right)\right)^{\prime} .
$$

By Theorem 1, there exists a solution $u_{2}$ of (7) satisfying $\alpha(t) \leq u_{2}(t)$ $\leq u_{1}(t)$ on $[0,1]$.

By induction, we can construct a nonincreasing sequence $\left\{u_{n}(t)\right\}$ such that

$$
\alpha(t) \leq u_{n}(t) \leq u_{n-1}(t) \leq \cdots \leq u_{0}(t)=\beta(t) .
$$


From condition $\left(\mathrm{H}_{4}\right)$, there exists a positive constant $N>0$ such that $\left|u_{n}(t)\right| \leq N, t \in I, n=1,2, \ldots$ On the other hand, $\left\{\left(\Phi_{m}\left(u_{n}^{\prime}\right)\right)^{\prime}\right\}$ is uniformly bounded on $I$ by equation (6). Therefore, $\left\{u_{n}\right\},\left\{\Phi_{m}\left(u_{n}^{\prime}\right)\right\}$ are uniformly bounded and equicontinuous. Applying the Arzelà-Ascoli theorem to the sequence $\left\{u_{n}\right\}$, we find that there exists a subsequence $\left\{u_{n_{k}}\right\}$ satisfying $\lim _{k \rightarrow \infty} \Phi_{m}\left(u_{n_{k}}^{\prime}\right)=v$. Thus, we obtain $\lim _{k \rightarrow \infty} u_{n_{k}}^{\prime}=\Phi_{m}^{-1}(v)$, and so

$$
u_{n_{k}}(t)=A+\int_{0}^{t} u_{n_{k}}^{\prime}(s) d s \rightarrow A+\int_{0}^{t} \Phi_{m}^{-1}(v) d s=\bar{u}(t) \quad(k \rightarrow \infty) .
$$

So there exists $\bar{u} \in C^{1}(I)$ such that $\lim _{k \rightarrow \infty} u_{n_{k}}(t)=\bar{u}(t)$. By the dominated convergence theorem, we know that $\bar{u}$ is a solution of problem (6).

TheOREm 3. Let $\alpha, \beta \in C^{1}[0,1]$ be lower and upper solutions of (1), respectively, with $\alpha \leq \beta$ on $I=[0,1]$. Assume that hypotheses $\left(\mathrm{H}_{3}\right)-\left(\mathrm{H}_{4}\right)$ are satisfied, and $g \in G, h \in H$. Then the boundary value problem (1)-(2) has a solution $u=u(t)$ with $\alpha(t) \leq u(t) \leq \beta(t)$ on $[0,1]$.

Proof. For each $\alpha(0) \leq c \leq \beta(0)$, there exists (by Theorem 2) a solution $u_{c}$ of the BVP

$$
\left(\Phi_{m}\left(u^{\prime}\right)\right)^{\prime}=f\left(t, u, T_{1} u, T_{2} u, u^{\prime}\right), \quad u(0)=c, \quad u(1)=h(c),
$$

satisfying $\alpha(t) \leq u_{c}(t) \leq \beta(t)$ on $[0,1]$. If $c=\alpha(0)$, then $u_{c}^{\prime}(0) \geq \alpha^{\prime}(0)$ and $u_{c}^{\prime}(1) \leq \alpha^{\prime}(1)$. Hence,

$$
\begin{aligned}
g\left(u_{c}(0), u_{c}(1), u_{c}^{\prime}(0), u_{c}^{\prime}(1)\right) & =g\left(\alpha(0), \alpha(1), u_{c}^{\prime}(0), u_{c}^{\prime}(1)\right) \\
& \geq g\left(\alpha(0), \alpha(1), \alpha^{\prime}(0), \alpha^{\prime}(1)\right) \geq 0
\end{aligned}
$$

by the monotonicity of $g$ in the last two variables. Similarly, if $c=\beta(0)$, we have $u_{c}^{\prime}(0) \leq \beta^{\prime}(0), u_{c}^{\prime}(1) \geq \beta^{\prime}(1)$, and therefore,

$$
\begin{aligned}
& g\left(u_{c}(0), u_{c}(1), u_{c}^{\prime}(0), u_{c}^{\prime}(1)\right) \leq g\left(\beta(0), \beta(1), \beta^{\prime}(0), \beta^{\prime}(1)\right) \leq 0, \\
& c=\beta(0) .
\end{aligned}
$$

Define

$$
\begin{aligned}
M & =\left\{c \in[\alpha(0), \beta(0)]: g\left(u_{c}(0), u_{c}(1), u_{c}^{\prime}(0), u_{c}^{\prime}(1)\right)<0\right\}, \\
N & =\left\{c \in[\alpha(0), \beta(0)]: g\left(u_{c}(0), u_{c}(1), u_{c}^{\prime}(0), u_{c}^{\prime}(1)\right)>0\right\} .
\end{aligned}
$$

If the theorem is not true, then $M \cup N=[\alpha(0), \beta(0)]$ and both $M, N$ are nonempty by (8)-(9). We claim that $M$ is closed. To see this, let $c_{n} \in M$ with $\lim _{n \rightarrow \infty} c_{n}=c_{0}$. Then with $u_{n}=u_{c_{n}}$, it follows that $g\left(u_{n}(0), u_{n}(1), u_{n}^{\prime}(0)\right.$, $\left.u_{n}^{\prime}(1)\right)<0$ and there exists a subsequence of $u_{n}$ which converges, uniformly on $[0,1]$, to a solution $u_{0}$ of (1) satisfying $u_{0}(0)=c_{0}, u_{0}(1)=h\left(c_{0}\right)$ and $g\left(u_{0}(0), u_{0}(1), u_{0}^{\prime}(0), u_{0}^{\prime}(1)\right) \leq 0$. By assumption, equality cannot occur, so that $g\left(u_{0}(0), u_{0}(1), u_{0}^{\prime}(0), u_{0}^{\prime}(1)\right)<0$, and thus $c_{0} \in M$. Therefore, $M$ is 
closed, so $N$ is open. Likewise, we may show $N$ is closed. This is a contradiction which proves the theorem.

THEOREM 4. Let $\alpha, \beta \in C^{1}[0,1]$ be lower and upper solutions of (1), respectively, with $\alpha \leq \beta$ on $I=[0,1]$. Assume that hypotheses $\left(\mathrm{H}_{3}\right)-\left(\mathrm{H}_{4}\right)$ are satisfied, and $p \in P, q \in Q$. Then the boundary value problem (1)-(3) has a solution $u=u(t)$ with $\alpha(t) \leq u(t) \leq \beta(t)$ on $[0,1]$.

THEOREM 5. Let $\alpha, \beta \in C^{1}[0,1]$ be lower and upper solutions of (1), respectively, with $\alpha \leq \beta$ on $I=[0,1]$. Assume that hypotheses $\left(\mathrm{H}_{3}\right)-\left(\mathrm{H}_{4}\right)$ are satisfied, and $r \in R, w \in W$. Then the boundary value problem (1)-(4) has a solution $u=u(t)$ with $\alpha(t) \leq u(t) \leq \beta(t)$ on $[0,1]$.

Corollary 1. Let $\alpha, \beta \in C^{1}[0,1]$ be lower and upper solutions of (1), respectively, with $\alpha \leq \beta$ on $I=[0,1]$. Assume that hypotheses $\left(\mathrm{H}_{3}\right)-\left(\mathrm{H}_{4}\right)$ are satisfied, and $m=(\beta(1)-\alpha(1)) /(\beta(0)-\alpha(0))$. Furthermore, suppose there exists $c>0$ such that $\beta^{\prime}(1)-\alpha^{\prime}(1) \geq c\left(\beta^{\prime}(0)-\alpha^{\prime}(0)\right)$ and let d satisfy $\beta^{\prime}(1)-c \beta^{\prime}(0) \geq d \geq \alpha^{\prime}(1)-c \alpha^{\prime}(0)$. Then equation (1) has a solution $u$ with $\alpha(t) \leq u(t) \leq \beta$ and $u(1)=m u(0)+\alpha(1)-m \alpha(0), u^{\prime}(1)=c u^{\prime}(0)+d$.

Corollary 2. Let $\alpha, \beta \in C^{1}[0,1]$ be lower and upper solutions of (1), respectively, with $\alpha \leq \beta$ on $I=[0,1]$. Suppose there exists a constant $L>0$ such that for all $(t, u) \in E$ and $u_{1}, u_{2} \in \mathbb{R}$,

$$
\left|f\left(t, u, T_{1} u, T_{2} u, u_{1}^{\prime}\right)-f\left(t, u, T_{1} u, T_{2} u, u_{2}^{\prime}\right)\right| \leq L\left|u_{1}^{\prime}-u_{2}^{\prime}\right| \cdot
$$

Let $A, B, a_{1}, a_{2}, b_{1}, b_{2}$ be real numbers such that $a_{i}, b_{i} \geq 0(i=1,2)$, $a_{1}+a_{2}>0, b_{1}+b_{2}>0$ and

$$
\begin{gathered}
a_{1} \alpha(0)-a_{2} \alpha^{\prime}(0)-A \leq 0 \leq a_{1} \beta(0)-a_{2} \beta^{\prime}(0)-A \\
b_{1} \alpha(1)+b_{2} \alpha^{\prime}(1)-B \leq 0 \leq b_{1} \beta(1)+b_{2} \beta^{\prime}(1)-B .
\end{gathered}
$$

Then equation (1) has a solution $u$ such that

$$
a_{1} u(0)-a_{2} u^{\prime}(0)-A=0=b_{1} u(1)+b_{2} u(1)-B, \quad \alpha(t) \leq u(t) \leq \beta(t) .
$$

ExAMPLE. To illustrate Theorem 4 for the case when the boundary conditions are nonlinear, let $f$ satisfy conditions $\left(\mathrm{H}_{3}\right)-\left(\mathrm{H}_{4}\right)$ and assume

$$
\begin{aligned}
& f(t,-1,\left.\psi_{1}(t)+\int_{0}^{t} K_{1}(t, s) d s, \psi_{2}(t)+\int_{0}^{t} K_{2}(t, s) d s, 0\right) \leq 0 \\
& \leq f\left(t, 1, \psi_{1}(t)+\int_{0}^{t} K_{1}(t, s) d s, \psi_{2}(t)+\int_{0}^{t} K_{2}(t, s) d s, 0\right), \quad 0 \leq t \leq 1,
\end{aligned}
$$

where $K_{i} \in C\left([0,1] \times[0,1], \mathbb{R}^{+}\right), \psi_{i} \in C([0,1], \mathbb{R}), i=1,2$, so that $\alpha=-1$, $\beta=1$ are lower and upper solutions, respectively, of $(1)$. Let $p=p(s, t)$, 
$q=q(s, t, u, v)$ be defined by

$$
p(s, t)=s^{2}-t-1, \quad q(s, t, u, v)=s+t+c u+d v,
$$

where $c \geq 5 / 4, d>0$ are real constants. It is easy to check that $p \in P$, $q \in Q$, so that by Theorem 4 , there exists a solution $u$ of the boundary value problem

$$
\begin{gathered}
\left(\Phi_{m}\left(u^{\prime}\right)\right)^{\prime}=f\left(t, u, T_{1} u, T_{2} u, u^{\prime}\right), \\
(u(0))^{2}-u^{\prime}(0)-1=0=u(0)+u^{\prime}(0)+c u(1)+d u^{\prime}(1),
\end{gathered}
$$

satisfying $-1 \leq u(x) \leq 1$ on $[0,1]$.

\section{References}

[1] A. Cabada and R. L. Pouso, Existence result for the problem $\left(\phi\left(u^{\prime}\right)\right)^{\prime}=f\left(t, u, u^{\prime}\right)$ with periodic and Neumann boundary conditions, Nonlinear Anal. 30 (1997), 1733-1742.

[2] - - - Existence result for the problem $\left(\phi\left(u^{\prime}\right)\right)^{\prime}=f\left(t, u, u^{\prime}\right)$ with nonlinear boundary conditions, ibid. 35 (1999), 221-231.

[3] C. De Coster, Pairs of positive solutions for the one-dimensional p-Laplacian, ibid. 23 (1994), 669-681.

[4] J. R. Esteban and J. L. Vázquez, On the equation of turbulent filtration in onedimensional porous media, ibid. 12 (1984), 1303-1325.

[5] D. J. Guo, Semi-Order Methods of Nonlinear Functional Analysis, Shandong Science and Technology Press, Jinan, 2000.

[6] M. A. Herrero and J. L. Vázquez, On the propagation properties of a nonlinear degenerate parabolic equation, Comm. Partial Differential Equations 7 (1982), 1381-1402.

[7] S. C. Hu and S. Leela, Periodic boundary value problem for integrodifferential equations of Hammerstein type, Appl. Math. Comput. 25 (1988), 29-38.

[8] D. O'Regan, Some general principles and results for $\left(\phi\left(u^{\prime}\right)\right)^{\prime}=q f\left(t, u, u^{\prime}\right), 0<t<1$, SIAM J. Math. Anal. 24 (1993), 648-668.

[9] - Existence theory for $\left(\phi\left(u^{\prime}\right)\right)^{\prime}=q f\left(t, u, u^{\prime}\right), 0<t<1$, Comm. Appl. Anal. 1 (1997), 33-52.

[10] H. S. Yang and Z. D. Yang, Existence of nonnegative solutions for singular nonlinear ordinary differential equations with nonlinear boundary conditions, Math. Appl. (Wuhan) 11 (1998), no. 3, 109-113 (in Chinese).

[11] Z. D. Yang, Two-point boundary value problems with nonlinear boundary conditions for a class of second order nonlinear ordinary differential equations, J. Math. Res. Exposition 15 (1995), no. 5, 7-12 (in Chinese).

[12] - Existence of positive solutions for a class of singular two-point boundary value problems of second order nonlinear equations, Appl. Math. Mech. 17 (1996), 465-476 (in Chinese).

[13] Z. D. Yang and Z. M. Guo, On the structure of positive solutions for quasilinear ordinary differential equations, Appl. Anal. 58 (1995), 31-51.

[14] - - - Existence of positive radial solutions and entire solutions for quasilinear singular boundary value problems, Ann. Differential Equations 12 (1996), 243-251 (in Chinese). 
[15] Z. D. Yang and H. S. Yang, Periodic boundary value problems for a class of quasilinear ordinary differential equations, ibid. 10 (1994), 464-470 (in Chinese).

School of Mathematics and Computer Science

Nanjing Normal University

Jiangsu, Nanjing, 210097, P. R. China

E-mail: zdyang_jin@263.net

Reçu par la Rédaction le 10.5.2004

Révisé le 30.10.2004 